\title{
Non-locality of the nucleon-nucleon potential from lattice QCD
}

Keiko Murano*

KEK, Tsukuba, Ibaraki 305-0801, Japan

E-mail: murano@het.ph.tsukuba.ac.jp

\section{Noriyoshi Ishii}

Department of Physics, The University of Tokyo, Tokyo 113-0033, Japan

\section{Sinya Aoki}

Graduate School of Pure and Applied Sciences, University of Tsukuba,

Tsukuba, Ibaraki 305-8571, Japan, and

Center for Computational Sciences, University of Tsukuba, Tsukuba, Ibaraki 305-8577, Japan

\section{Tetsuo Hatsuda}

Department of Physics, The University of Tokyo, Tokyo 113-0033, Japan, and

Institute for the Physics and Mathematics of the Universe (IPMU), The University of Tokyo,

Chiba 277-8568, Japan

\section{for HAL QCD Collaboration}

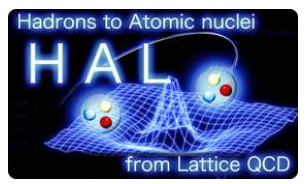

The Nambu-Bethe-Salpeter (NBS) wave function for two nucleons on the lattice has been shown to yield a non-local and energy-independent nucleon-nucleon $(\mathrm{NN})$ potential, $U\left(\vec{r}, \vec{r}^{\prime}\right)$. In practice, the derivative expansion of $U\left(\vec{r}, \vec{r}^{\prime}\right)$ is currently employed to determine the potential at low energies. In this report, we study the magnitude of non-locality to check the convergence of such a derivative expansion. With quenched lattice QCD at $m_{\pi}=530 \mathrm{MeV}$, we compare the NN potentials at the center of mass energy $E \simeq 0 \mathrm{MeV}$ and at $E \simeq 45 \mathrm{MeV}$. We also investigate the angular momentum dependence of the spin singlet potential, by comparing the potentials in ${ }^{1} S_{0}$ and ${ }^{1} D_{2}$ channels. We find that the non-locality and the angular momentum dependence in the above energy range are negligible within statistical errors.

The XXVIII International Symposium on Lattice Field Theory, Lattice2010

June 14-19, 2010

Villasimius, Italy

\footnotetext{
${ }^{*}$ Speaker.
} 


\section{Introduction}

The nucleon-nucleon $(\mathrm{NN})$ potential, which describes the interaction between two nucleons at low energies, is widely used as a basic tool to study the structures and reactions of atomic nuclei. In the past few decades, several realistic NN potentials have been proposed: These are phenomenologically determined so as to reproduce the NN scattering phases of more than 4000 data points with $\chi^{2} / N_{\text {dof }} \sim 1$ [1, 2, 3]. Recently a first-principle method to extract the NN potential from the Nambu-Bethe-Salpeter (NBS) wave function in lattice QCD has been proposed by three of the present authors [4, 5]. This method can be generalized also to derive the hyperon potentials (YN and YY) [6, 7] and the three-nucleon potentials [8], which do not have enough experimental data. The potentials based on lattice QCD, once obtained in good accuracy, would become firstprinciple inputs to the studies of ordinary nuclei, hyper nuclei, and the interior of the neutron stars by using advanced techniques in nuclear many-body problems.

The potential obtained from the NBS wave function in lattice QCD simulations is non-local and energy-independent, $U\left(\vec{r}, \vec{r}^{\prime}\right)$. To determine the potential at low energies, we have proposed to make a derivative expansion of $U\left(\vec{r}, \vec{r}^{\prime}\right)$ and calculate the local coefficient functions successively [5]. The aim of this report is to examine the convergence of such a derivative expansion. In sec.2. we give a brief review of our method to construct the non-local potential and its derivative expansion. In sec.\}, we show that the non-locality of $U\left(\vec{r}, \vec{r}^{\prime}\right)$ can be checked by the center of mass energy $(E)$ dependence and the orbital angular momentum $(L)$ dependence of a "truncated local potential", $V^{\text {trun }}(r ; E, L)$. In sec. 7 , we show our numerical results of $V^{\operatorname{trun}}(r ; E, L=0)$ at two different scattering energies, $E \simeq 0 \mathrm{MeV}$ and $45 \mathrm{MeV}$. In addition we examine the $L$ dependence of the truncated local potential in the two spin-singlet channels, ${ }^{1} S_{0}$ and ${ }^{1} D_{2}$. We find negligible energy and angular momentum dependence of $V^{\operatorname{trun}}(r ; E, L)$, which implies that the non-locality $U\left(\vec{r}, \vec{r}^{\prime}\right)$ is small at low energies up to $E \simeq 45 \mathrm{MeV}$.

\section{NN potential from Lattice QCD and its derivative expansion}

The equal-time NBS wave function ${ }^{1}$ is defined as

$$
\phi(\vec{r}, k) \equiv \frac{1}{V} \sum_{\vec{x}}\langle 0|n(\vec{x}+\vec{r}) p(\vec{x})| B=2 ; k\rangle,
$$

where " $k$ " is the "asymptotic momentum" related to the total energy as $W=2 \sqrt{m_{N}^{2}+k^{2}}$. Also, the local composite operators for the nucleon are defined as $n_{\beta}(y)=\varepsilon_{a b c}\left(u_{a}(y) C \gamma_{5} d_{b}(y)\right) d_{c \beta}(y)$ and $p_{\alpha}(x)=\varepsilon_{a b c}\left(u_{a}(x) C \gamma_{5} d_{b}(x)\right) u_{c \alpha}(x)$, where $a, b, c$ denote color indices, and $C$ is the charge conjugation matrix. It has been discussed that $\phi(\vec{r}, k)$ below the pion production threshold satisfies the Schrödinger-type equation [曰, 5]:

$$
\left(\triangle+k^{2}\right) \phi(\vec{r} ; k)=2 \mu \int d^{3} r^{\prime} U\left(\vec{r}, \vec{r}^{\prime}\right) \phi\left(\vec{r}^{\prime}, k\right),
$$

which defines the non-local potential and $k$-independent potential $U\left(\vec{r}, \vec{r}^{\prime}\right)$ with $\mu \equiv m_{N} / 2$ being the reduced mass. By using the Nishijima-Zimmermann-Haag reduction formula for composite

\footnotetext{
${ }^{1}$ The NBS wave function was also utilized to investigate Lüscher's finite volume method for the pion-pion scattering phase shift in Ref.[10, 11].
} 
operators, it can be shown that, for large $|\vec{r}|, \phi(\vec{r} ; k)$ has an asymptotic form characterized by the scattering phase $\delta(k)[5]$ :

$$
\phi(\vec{r} ; k) \sim \frac{\sin (k r+\delta(k))}{k r}+\cdots .
$$

This functional form is exactly the same as the asymptotic form of the scattering wave in the non-relativistic quantum mechanics. Therefore our non-local potential $U\left(\vec{r}, \vec{r}^{\prime}\right)$ is to reproduce the scattering phase $\delta(k)$ below the pion production threshold.

Although the non-local potential $U\left(\vec{r}, \vec{r}^{\prime}\right)$ has nice formal properties, its explicit construction is not easy in lattice QCD simulations, where the number of available NBS wave functions for different scattering energies are limited. Therefore, we employ the derivative expansion as shown below and determine the local coefficient functions successively by using low-energy NBS wave functions:

$$
U\left(\vec{r}, \vec{r}^{\prime}\right)=[\underbrace{V_{0}^{I}(r)+V_{\sigma}^{I}(r)\left(\sigma_{1} \cdot \sigma_{2}\right)+V_{T}^{I}(r) S_{12}}_{L O}+\underbrace{V_{L S}^{I}(r) \vec{L} \cdot \vec{S}}_{N L O}+\mathscr{O}\left(\vec{\nabla}^{2}\right)] \delta\left(\vec{r}-\vec{r}^{\prime}\right) .
$$

Here $I=0,1$ denotes the total isospin of the two nucleons and $\vec{S} \equiv\left(\vec{\sigma}_{1}+\vec{\sigma}_{2}\right) / 2$ is the total spin operator. Also, the tensor operator is written as $S_{12} \equiv 3\left(\vec{\sigma}_{1} \cdot \vec{r}\right)\left(\vec{\sigma}_{2} \cdot \vec{r}\right) / r^{2}-\vec{\sigma}_{1} \cdot \vec{\sigma}_{2}$. The LO terms such as the central potential in the ${ }^{1} S_{0}$ channel, and the central and the tensor potentials in the ${ }^{3} S_{1}-{ }^{3} D_{1}$ channel have been obtained so far at CM energy $E \sim 0 \mathrm{MeV}$ (see e.g. a recent summary [9, 51).

\section{Energy and angular momentum dependence as a test of non-locality}

In this section we discuss that the convergence of the derivative expansion of $U\left(\vec{r}, \vec{r}^{\prime}\right)$ in the NN system can be examined by studying the energy and the angular momentum dependence of the truncated local potential at finite $E .{ }^{2}$ For simplicity, we restrict ourselves to the spin-singlet case $(S=0)$ where $S_{12}$ and $\vec{L} \cdot \vec{S}$ do not contribute. Then we obtain

$$
\left(\Delta+k^{2}\right) \phi^{S=0}(\vec{r} ; k)=2 \mu\left[V_{\mathrm{C}}(r)+\left\{\nabla^{2}, V_{p^{2}}(r)\right\}+V_{L^{2}}(r) L^{2}+\cdots\right] \phi^{S=0}(\vec{r} ; k),
$$

where $V_{\mathrm{C}}(r) \equiv V_{0}(r)-3 V_{\sigma}(r)$ is the LO potential in the derivative expansion, while the higher order terms with $L^{2 n}, \nabla^{2 n}$ are referred to $\mathrm{N}^{2 n} \mathrm{LO}$ terms. If we have independent NBS wave functions for different energies and angular momenta, we can determine $\mathrm{N}^{2 n} \mathrm{LO}$ terms order by order starting from a small $n$. For such a procedure making sense, the magnitude of the potential at the leading order should be dominant at low energies and low angular momenta. A simplest way to check this is to define a "truncated local potential" given below and study its $E$ and $L$ dependence:

$$
V_{\mathrm{C}}^{\mathrm{trun}}(r ; E, L) \equiv \frac{1}{2 \mu} \frac{\nabla^{2} \phi^{S=0}(\vec{r} ; k)}{\phi^{S=0}(\vec{r} ; k)}+\frac{k^{2}}{2 \mu} .
$$

If $V_{\mathrm{C}}^{\text {trun }}(r ; E, L)$ has small $E$ and $L$ dependence, it is a good approximation of $V_{\mathrm{C}}(r)$. On the other hand, if it has large $E$ and/or $L$ dependence, one cannot neglect $V_{p^{2}}(r), V_{L^{2}}(r)$ etc, so that they must be determined together with $V_{\mathrm{C}}(r)$ by using the NBS wave functions for several different values of $E$ and $L$.

\footnotetext{
${ }^{2}$ In the Ising field theory, it is analytically shown that the energy-dependence is weak at low energy, indicating that the non-locality of the potential is weak [12].
} 


\section{Numerical Simulations and results}

\subsection{Lattice QCD setup}

We employ the standard plaquette gauge action on a $32^{3} \times 48$ lattice at $\beta=5.7$ for quenched gauge configurations. Quark propagators are calculated with the standard Wilson quark action at $\kappa=0.1665$. This setup gives the lattice spacing $a^{-1}=1.44(2) \mathrm{GeV}(a \simeq 0.137 \mathrm{fm})$ from $m_{\rho}$ input, the spatial extension $L=32 a \simeq 4.4 \mathrm{fm}, m_{\pi} \simeq 0.53 \mathrm{GeV}$ and $m_{N} \simeq 1.33 \mathrm{GeV}$ [13]. Quenched gauge configurations are generated by the heat bath algorithm with over relaxations. Potentials are measured with configurations separated by 200 sweep, and 4000 configurations are accumulated to obtain results in this report. These calculations are performed on Blue Gene/L at KEK.

The NBS wave function is obtained from the nucleon four-point correlator in the large $t$ region,

$$
\begin{aligned}
G^{(4)}\left(\vec{x}, \vec{y}, t, t_{0}\right) & =\left\langle 0\left|n(\vec{y}, t) p(\vec{x}, t) \overline{\mathscr{J}}_{p n}\left(t_{0}\right)\right| 0\right\rangle=\sum_{n} A_{n}\left\langle 0|n(\vec{y}) p(\vec{x})| B=2 ; W_{n}\right\rangle e^{-W_{n}\left(t-t_{0}\right)} \\
& \rightarrow A_{0}\left\langle 0|n(\vec{y}) p(\vec{x})| B=2 ; W_{0}\right\rangle e^{-W_{0}\left(t-t_{0}\right)}
\end{aligned}
$$

where $W_{n}=2 \sqrt{m_{N}^{2}+k_{n}^{2}}$ and $A_{n} \equiv\left\langle B=2 ; W_{n}\left|\overline{\mathscr{J}}_{p n}\right| 0\right\rangle$. Here $\overline{\mathscr{J}}_{p n}\left(t_{0}\right) \equiv \bar{P}\left(t_{0}\right) \bar{N}\left(t_{0}\right)$ is a source operator located at $t=t_{0}$ with $\bar{P}_{\alpha} \equiv \varepsilon_{a, b, c}\left(\bar{U}_{a} C \gamma_{5} \bar{D}_{b}\right) \bar{U}_{c \alpha}$ and $\bar{N}_{\beta} \equiv \varepsilon_{a, b, c}\left(\bar{U}_{a} C \gamma_{5} \bar{D}_{b}\right) \bar{D}_{c \beta}$. Up and down quark operators associated with a source function $f(x)$ specified later are denoted as $U(t) \equiv$ $\sum_{\vec{x}} u(t, \vec{x}) f(\vec{x})$ and $D(t) \equiv \sum_{\vec{x}} d(t, \vec{x}) f(\vec{x})$. By examining the $t$ dependence of potentials, we confirm that the ground state saturation for potentials is achieved at $t-t_{0} \geq 9$.

\subsection{Details of the calculation}

The NN potentials at $\mathrm{CM}$ energy $E \equiv k^{2} /(2 \mu) \simeq 0 \mathrm{MeV}$ with $\mu=m_{N} / 2$ is constructed by using the periodic boundary condition (PBC), which is imposed on the quark fields along spatial directions. Since spatial momentum of each quark is discretized as $p_{i} \simeq 2 \pi n_{i} / L\left(n_{i} \in \mathbb{Z}\right)$ in the PBC, the ground state corresponds to $p_{i} \simeq 0$. For the PBC, we employ the wall source, i.e., $f(\vec{r})=1$ with the Coulomb gauge fixing only at $t=t_{0}$ to enhance the overlap with the ground state. The wall source provides us with the $A_{1}^{+}$orbital contribution, by which we can only study S-wave $(L=0)$ for the spin singlet case with possible contaminations from higher partial waves with $L \geq 4$. To improve the statistics, we calculate the NBS wave function four times on each configuration by putting the source point at four different time slices.

The NN potentials at $\mathrm{CM}$ energy $E \neq 0$ is constructed by using the anti-periodic boundary condition (APBC), which is imposed on the quark fields along spatial directions. Since the spatial momentum of each quark is discretized as $p_{i} \simeq(2 n+1) \pi / L\left(n_{i} \in \mathbb{Z}\right)$ in the APBC, the ground state corresponds to $p_{i} \simeq \pm \pi / L$, whose energy is given by $E \simeq 3 \times(\pi / L)^{2} /(2 \mu) \simeq 45 \mathrm{MeV}$ for $L \simeq 4.4 \mathrm{fm}$. For the APBC, we employ four types of "momentum-wall" sources, i.e., $f(\vec{r}) \equiv$ $\cos ((x+y+z) \pi / L), \cos ((-x+y+z) \pi / L), \cos ((x-y+z) \pi / L)$, and $\cos ((-x-y+z) \pi / L)$ with the Coulomb gauge fixing only at $t_{0}$ to enhance the overlap with the ground state with $p_{i} \sim \pm \pi / L$. In addition to the $A_{1}^{+}$orbital contribution, our momentum-wall source provides us with the $T_{2}^{+}$ orbital contribution, which makes it possible to study D-wave $(L=2)$ for spin singlet case with possible contaminations from higher partial waves with $L \geq 4$. 
The NBS wave functions for the S-wave and the D-wave are extracted by projection operators,

$$
\phi(\vec{r} ; k ; \Gamma)=\frac{d_{\Gamma}}{24} \sum_{i=0}^{23} \chi^{(\Gamma)}\left(R_{i}\right)^{*} \phi^{S=0}(R \cdot \vec{r} ; k),
$$

where $\chi^{(\Gamma)}$ denotes the character of the representation $\Gamma$ for the cubic group $O$ (See Table.1), $R_{i}$ denotes one of the 24 elements of the cubic group, and $d_{\Gamma}$ denotes the dimension of the representation, i.e., $d_{A 1}=1$ and $d_{T 2}=3$. The calculation performed with $\Gamma=A_{1}$ in the PBC provides us with the NBS wave function for ${ }^{1} S_{0}$ at $E \simeq 0 \mathrm{MeV}$, while the calculation with $\Gamma=A_{1}$ and $T_{2}$ in the APBC provides us with NBS wave functions for ${ }^{1} S_{0}$ and ${ }^{1} D_{2}$ at $E \simeq 45 \mathrm{MeV}$, respectively. These NBS wave functions are inserted into eq.(3.2) to obtain $V_{\mathrm{C}}^{\mathrm{trun}}(r ; E, L)$, where $k^{2} / 2 \mu$ in this report is estimated from the non-interacting nucleons on the lattice, i.e., $k^{2} / 2 \mu=0 \mathrm{MeV}$ for the PBC, and $k^{2} / 2 \mu=3(\pi / L)^{2} / 2 \mu=45 \mathrm{MeV}$ for the APBC.

\subsection{Results}

In the top panel of Fig. 11, we compare $V_{\mathrm{C}}^{\mathrm{trun}}(r ; E, L=0)$ in the ${ }^{1} S_{0}$ channel obtained at $E \simeq 45$ $\mathrm{MeV}$ (red) with that at $E \simeq 0 \mathrm{MeV}$ (blue). All data are taken at $t-t_{0}=9$, where the ground state saturation is numerically confirmed as mentioned before. We observe that the agreement of the potentials between the two energies is quite good within statistical errors. The comparison of $V_{\mathrm{C}}^{\mathrm{trun}}(r ; E, L)$ in the ${ }^{1} S_{0}(L=0)$ channel (red) with that in the ${ }^{1} D_{2}(L=2)$ channel (cyan) obtained at $E \simeq 45 \mathrm{MeV}$ is shown in the bottom panel of Fig. 11. We observe that the angular momentum dependence of $V_{\mathrm{C}}^{\mathrm{trun}}(r ; E, L)$ is weak, though the statistical errors are rather large in the ${ }^{1} D_{2}$ channel.

By these comparisons, we find that the energy and the angular momentum dependence of $V_{\mathrm{C}}^{\text {trun }}(r ; E, L)$ is very weak within statistical errors. We therefore conclude that contributions from higher order terms in the derivative expansion are small and that the LO local potential in the derivative expansion is the good approximation for the non-local potential $U\left(\vec{r}, \vec{r}^{\prime}\right)$ at least up to the energy $E \simeq 45 \mathrm{MeV}$ and angular momentum $L \leq 2$.

\section{Summary and discussion}

We have studied the validity of the derivative expansion for the non-local NN potential $U\left(\vec{r}, \vec{r}^{\prime}\right)$ in quenched QCD at $m_{\pi} \simeq 530 \mathrm{MeV}$. The convergence is tested by the energy and the angular

\begin{tabular}{|c||ccccc|}
\hline$\Gamma$ & $\mathrm{E}$ & $6 C_{4}$ & $3 C_{2}$ & $8 C_{3}$ & $6 C_{2}$ \\
\hline$A_{1}$ & 1 & 1 & 1 & 1 & 1 \\
$A_{2}$ & 1 & -1 & 1 & 1 & -1 \\
$E$ & 2 & 0 & 2 & -1 & 0 \\
$T_{1}$ & 3 & 1 & -1 & 0 & -1 \\
$T_{2}$ & 3 & -1 & -1 & 0 & 1 \\
\hline
\end{tabular}

Table 1: The character table of the cubic group $O$. The notations of classes $\left(E, 6 C_{4}, \cdots\right)$ and irreducible representation $\left(A_{1}, A_{2}, \cdots\right)$ follow Mulliken Symbols. 

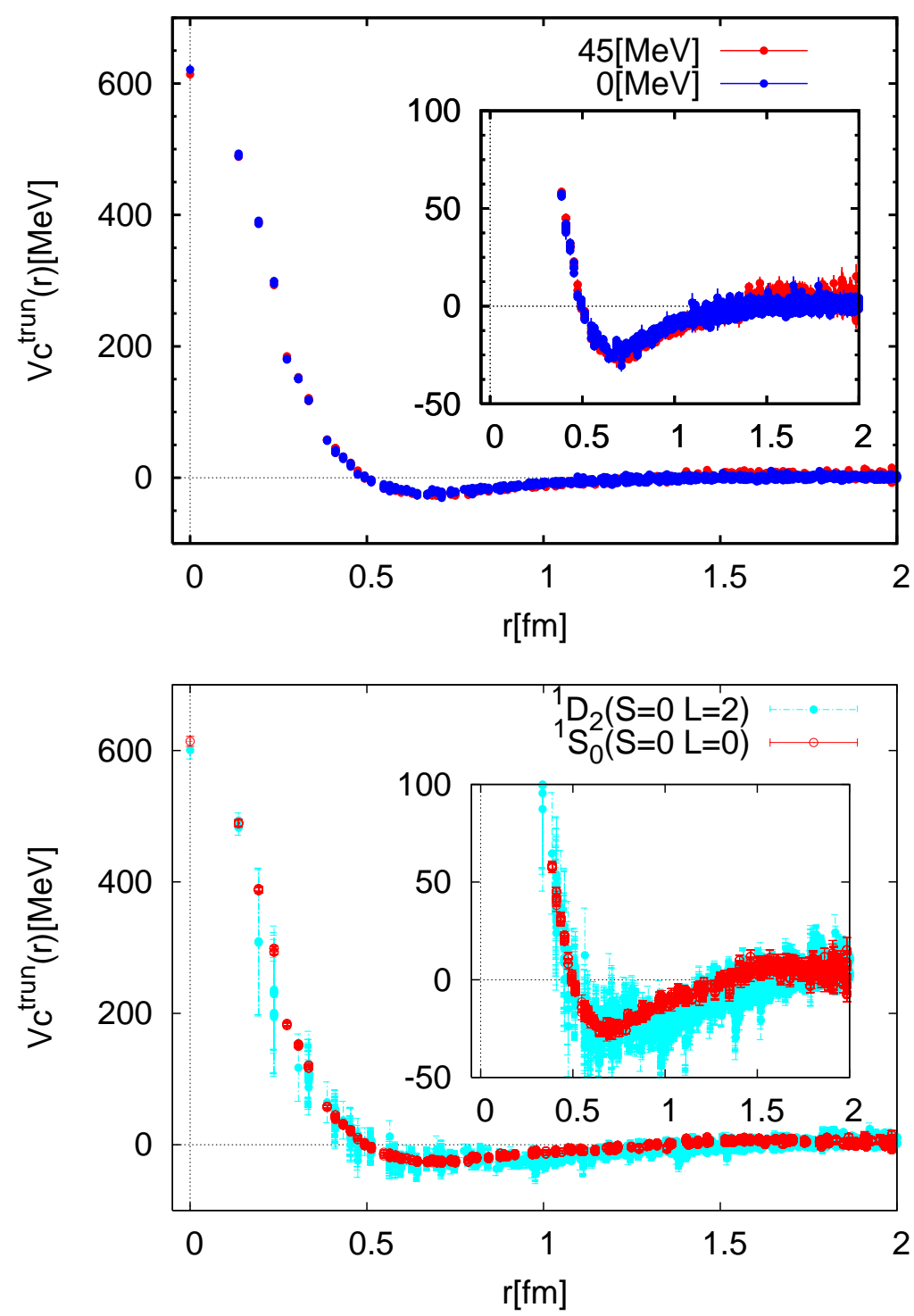

Figure 1: Top: A comparison of the truncated local potential $V_{\mathrm{C}}^{\text {trun }}(r ; E, L=0)$ in the ${ }^{1} S_{0}$ channel with PBC (blue) and that with APBC (red) at $t-t_{0}=9$ and $r \leq 2.0 \mathrm{fm}$. Bottom: A comparison of $V_{\mathrm{C}}^{\text {trun }}(r ; E, L)$ with the APBC for the ${ }^{1} S_{0}$ channel (red) and that for the ${ }^{1} D_{2}$ channel (cyan).

momentum dependence of the truncated local potential $V_{\mathrm{C}}^{\text {trun }}(r ; E, L)$ : To examine the energy dependence, we have generated $V_{\mathrm{C}}^{\mathrm{trun}}(r ; E, L=0)$ in the ${ }^{1} S_{0}$ channel at two different energies, $E \simeq 0$ $\mathrm{MeV}$ and $E \simeq 45 \mathrm{MeV}$. We have found that the energy dependence of the truncated potential is very weak. We have also generated $V_{\mathrm{C}}^{\text {trun }}(r ; E, L=2)$ for ${ }^{1} D_{2}$ at $E \simeq 45 \mathrm{MeV}$ in the same setup. From the comparison with $V_{\mathrm{C}}^{\text {trun }}(r ; E, L=0)$ at $E \simeq 45 \mathrm{MeV}$, we have observed that the angular momentum dependence of the effective potential is also small. These results indicate that the LO potentials constructed from NBS wave functions obtained at $E \simeq 0 \mathrm{MeV}$ can be safely used for $0 \leq E \leq 45 \mathrm{MeV}$ and the angular momentum $L \leq 2$. 
An extension of the current analysis to NN potentials in the spin-triplet $(S=1)$ channel is ongoing. It is an important future task to perform the same analysis for other baryon-baryon potentials and for the potentials in full QCD with lighter pion masses. Eventually, we need to extract NLO terms such as the spin-orbit potential from the NBS wave functions with finite angular momentum on the lattice.

\section{Acknowledgement}

We are grateful for authors and maintainers of CPS++[14], a modified version of which is used for simulation done in this work. Numerical calculations in this study have been performed under the Large Scale Simulation Program No.0923(FY2009) of High Energy Accelerator Research Organization (KEK). This work is supported in part by Grant-in-Aid of the Ministry of Education, Science and Technology, Sports and Culture (Nos. 20340047, 20105001, 20105003, 22540268, 19540261, ).

\section{References}

[1] R. Machleidt, Phys. Rev. C 63, 024001 (2001) [arXiv:nucl-th/0006014].

[2] R. B. Wiringa, V. G. J. Stoks and R. Schiavilla, Phys. Rev. C 51, 38 (1995) [arXiv:nucl-th/9408016].

[3] V. G. J. Stoks, R. A. M. Klomp, C. P. F. Terheggen and J. J. de Swart, Phys. Rev. C 49, 2950 (1994) [arXiv:nucl-th/9406039].

[4] N. Ishii, S. Aoki and T. Hatsuda, Phys. Rev. Lett. 99, 022001 (2007) [arXiv:nucl-th/0611096].

[5] S. Aoki, T. Hatsuda and N. Ishii, Prog. Theor. Phys. 123, 89 (2010) [arXiv:0909.5585 [hep-lat]].

[6] H. Nemura, N. Ishii, S. Aoki and T. Hatsuda, Phys. Lett. B 673, 136 (2009) [arXiv:0806.1094 [nucl-th]].

[7] T. Inoue et al. [HAL QCD collaboration], Prog. Theor. Phys. 124, 591 (2010) [arXiv:1007.3559 [hep-lat]].

[8] T. Doi for HAL QCD Collaboration, arXiv:1011.0657 [hep-lat].

[9] N. Ishii [PACS-CS Collaboration and HAL-QCD Collaboration], PoS LAT2009, 019 (2009) [arXiv:1004.0405 [hep-lat]].

[10] C. J. D. Lin, G. Martinelli, C. T. Sachrajda and M. Testa, Nucl. Phys. B 619, 467 (2001) [arXiv:hep-lat/0104006].

[11] S. Aoki et al. [CP-PACS Collaboration], Phys. Rev. D 71, 094504 (2005) [arXiv:hep-lat/0503025].

[12] S. Aoki, J. Balog and P. Weisz, Prog. Theor. Phys. 121, 1003 (2009) [arXiv:0805.3098 [hep-th]].

[13] M. Fukugita, Y. Kuramashi, M. Okawa, H. Mino and A. Ukawa, Phys. Rev. D 52, 3003 (1995) [arXiv:hep-lat/9501024].

[14] Columbia Physics System (CPS) http://qcdoc.phys.columbia.edu/cps.html. 